Van $\mathrm{Vl}$ eck par amagnet i sm of the thul i um gar net TmBAl 5012

\begin{tabular}{|l|l|}
\hline 著者 & AWAKA Junj i, ENDO Ryo, NAGATA Shoi chi \\
\hline $\begin{array}{l}\text { j our nal or } \\
\text { publ i cat } \mathrm{i} \text { on } \mathrm{t} \text { i t l e }\end{array}$ & Journal of physi cs and chemi stry of sol i ds \\
\hline vol une & 64 \\
\hline number & 12 \\
\hline page $r$ ange & $2403-2408$ \\
\hline year & $2003-12$ \\
\hline URL & ht t p: //hdl . handl e. net /10258/350 \\
\hline
\end{tabular}




\title{
Van Vleck paramagnetism of the thulium garnet $\mathrm{Tm}_{3} \mathrm{Al}_{5} \mathrm{O}_{12}$
}

\author{
Junji Awaka, Ryo Endoh, Shoichi Nagata* \\ Department of Materials Science and Engineering, Muroran Institute of Technology, \\ 27-1 Mizumoto-cho, Muroran, Hokkaido, 050-8585 Japan
}

\begin{abstract}
The magnetic susceptibility of the garnet-type single crystal $\mathrm{Tm}_{3} \mathrm{Al}_{5} \mathrm{O}_{12}$ exhibits the typical Van Vleck temperature independent paramagnetism below $\approx 8 \mathrm{~K}$. The temperature dependence of the susceptibility over the range 2.0 to $300 \mathrm{~K}$ has been analyzed on the assumption that the cubic crystal-electric-field dominates the energy level on ${ }^{3} \mathrm{H}_{6}(J=6)$ ground multiplet for $\mathrm{Tm}^{3+}$ ion having 12-electrons in $4 f$ shell. The ground state of the ${ }^{3} \mathrm{H}_{6}$ is nonmagnetic with $\Gamma_{2}$ singlet, avoiding the Kramers doublet. The energy separation between $\Gamma_{2}$ and the first excited state $\Gamma_{5}^{(2)}$ triplet is evaluated to be $68.0 \mathrm{~K}$. The whole energy interval $\Delta$ between $\Gamma_{2}$ and the highest state $\Gamma_{1}$ in ${ }^{3} \mathrm{H}_{6}$ is estimated to be $339.5 \mathrm{~K}$.
\end{abstract}

Key words: A. Oxides; D. Magnetic properties; D. Crystal fields

*Corresponding author. Fax: +81-143-46-5612.

E-mail address: naga-sho@mmm.muroran-it.ac.jp (S. Nagata) 


\section{Introduction}

An ion which is occupied with an even number of $f$-electrons is a candidate for the ground state singlet in the energy level, avoiding the Kramers doublet. The Van Vleck temperature independent paramagnetic susceptibility originates from these conditions. We have grown the single crystal of garnet-structure $\mathrm{Tm}_{3} \mathrm{Al}_{5} \mathrm{O}_{12}$ and measured magnetic susceptibility over a temperature range 2.0 to $300 \mathrm{~K}$. The Van Vleck paramagnetic susceptibility has been found. The theoretical analysis for the susceptibility has been made on assuming a simplified cubic crystal field symmetry with one unknown parameter. It should be noted that the actual symmetry for the $\mathrm{Tm}^{3+}$ ion decreases to $D_{2}$ orthorhombic with local distortion, however nine unknown crystal field parameters have not yet been determined.

The $\mathrm{Tm}^{3+}$ ion has an even number of 12 -electrons in $4 f$ shell, avoiding the Kramers doublets. Therefore, the energy level of $\mathrm{Tm}^{3+}$ ion may be that of ground state singlet in the crystal-electric-field (CEF). If the CEF ground state is singlet, $\left\langle 0\left|J_{z}\right| 0\right\rangle=0$, then no effect of external magnetic field is expected within the first approximation. However, second order perturbation theory predicts a change in the ground state energy because it takes account of excited states with $<_{s}\left|J_{z}\right| s>\neq 0$ being mixed in. The magnetic susceptibility shows the temperature independent behavior at low temperature, known as the Van Vleck paramagnetism. We present in this article a typical Van Vleck paramagnetism of the garnet-type $\mathrm{Tm}_{3} \mathrm{Al}_{5} \mathrm{O}_{12}$. In the previous reports $[1,2]$, the only low temperature data (below $20 \mathrm{~K}$ ) of the susceptibility for $\mathrm{Tm}_{3} \mathrm{Al}_{5} \mathrm{O}_{12}$ have been given. These data were less accurate. A re-investigation of the magnetism for $\mathrm{Tm}_{3} \mathrm{Al}_{5} \mathrm{O}_{12}$ has been made in the present study. We have measured the susceptibility over the wide range 2.0 - $300 \mathrm{~K}$, and show the manifest Van Vleck paramagnetic susceptibility at low temperature.

Thulium aluminum garnet $\mathrm{Tm}_{3} \mathrm{Al}_{5} \mathrm{O}_{12}$ has the garnet structure of cubic symmetry of space group $I a \overline{3} d\left(\mathrm{O}_{\mathrm{h}}{ }^{10}\right)$ as shown in Figs. 1 and 2. The general chemical formula for an oxide garnet may be written as $\{C\}_{3}[A]_{2}(D)_{3} \mathrm{O}_{12}$, with eight of these formula units per unit cell, where the three cation sites are $C$ (dodecahedral), $A$ (octahedral), and $D$ (tetrahedral)-sites in the garnet $[3,4]$. These sites are surrounded by oxygen ions $\mathrm{O}^{2-}$. Generally, larger ions occupy in order of $C, A$, and $D$-site. $\mathrm{Tm}^{3+}$ ions occupy the $C$-site, $\mathrm{Al}^{3+}$ ions occupy the $A$ and $D$-sites, where the magnetic ion is the $\mathrm{Tm}^{3+}$ ion.

\section{Experimental methods}

Single crystals of garnet-type $\mathrm{Tm}_{3} \mathrm{Al}_{5} \mathrm{O}_{12}$ have been grown by a flux method $[5,6]$. The dc magnetic susceptibility was measured with a Quantum Design superconducting quantum interference device ( $r f$-SQUID) magnetometer over the temperature range $2.0-300 \mathrm{~K}$ in a constant magnetic field of $10 \mathrm{kOe}$. 


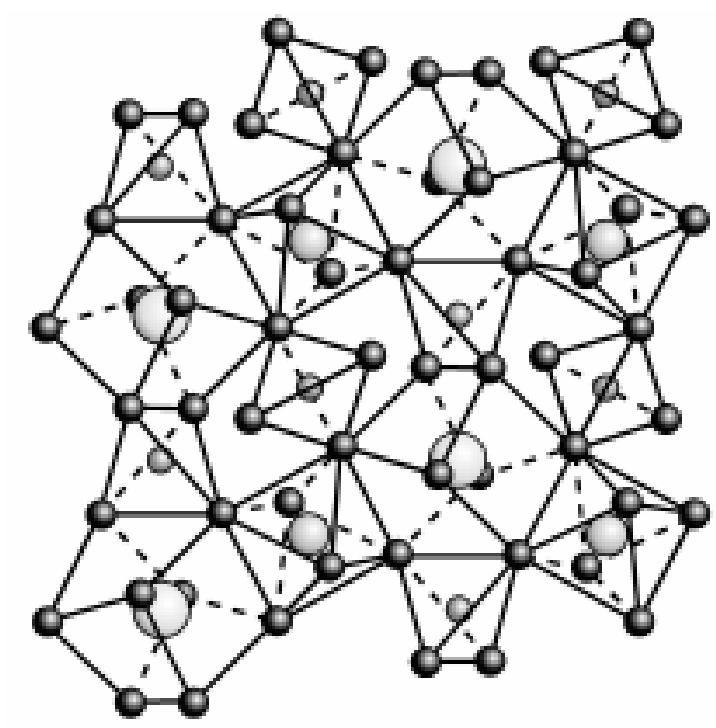

General Chemical Formula : $\{C\}_{3}[A]_{2}(D)_{3} \mathrm{O}_{12}$
$\bigcirc:\{C\}$
$\bigcirc:[A]$
$0:(D)$
: $\mathrm{O}$

Fig. 1. Garnet structure of cubic symmetry with space group $\operatorname{Ia}_{3}^{-} d\left(\mathrm{O}_{\mathrm{h}}{ }^{10}\right)$.

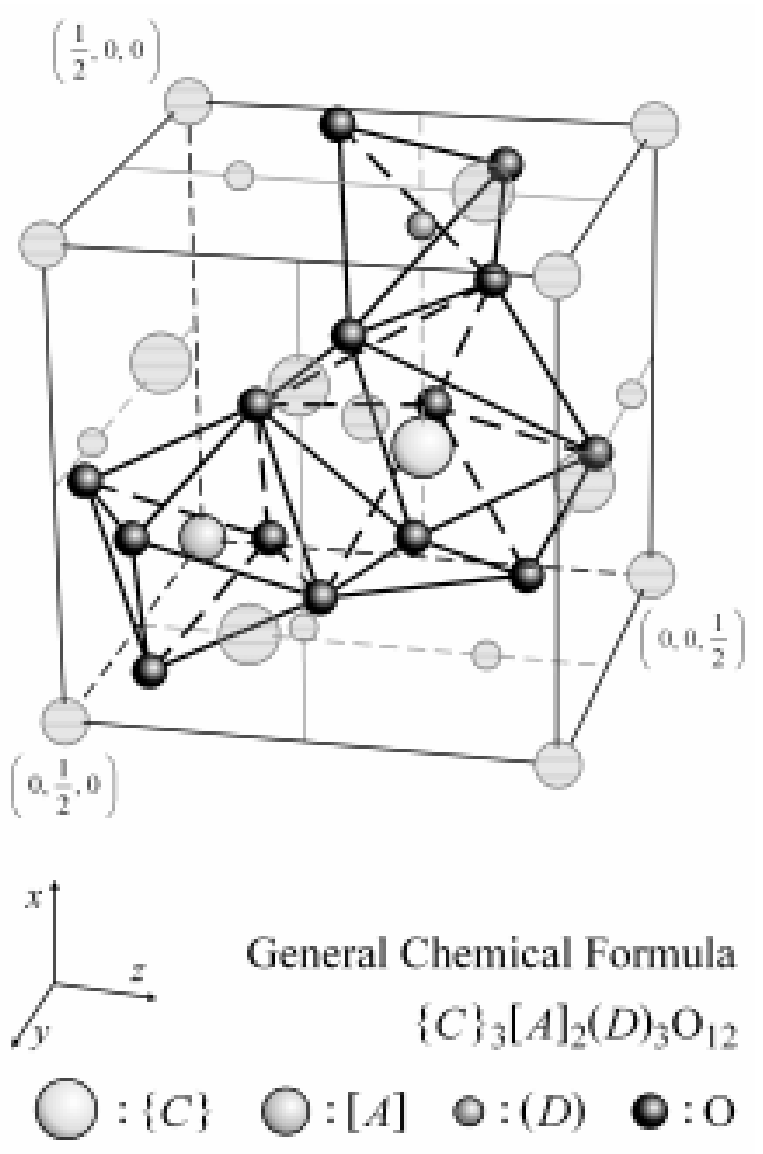

Fig. 2. Garnet structure with the different sites $\{C\},[A]$ and $(D)$. The space lattice is $b c c$, and the $x-y-z$ coordinates of these sites are $C$-site at $1 / 801 / 4, A$-site at 000 and $D$-site at 3/8 $01 / 4$. 


\section{Results and discussion}

The grown crystals of $\mathrm{Tm}_{3} \mathrm{Al}_{5} \mathrm{O}_{12}$ have a maximum edge of about $2-3 \mathrm{~mm}$ in size with shining, light green surface. The lattice constant is $11.960 \AA$ at room temperature.

The magnetic susceptibility exhibits the temperature independent Van Vleck paramagnetism below $\approx 8 \mathrm{~K}$, as shown in Fig. 3. The susceptibility at low temperature indicates that the ground state of $\mathrm{Tm}^{3+}$ ion is non-magnetic state under the influence of CEF. The detailed analysis has been made on the assumption that the influence of dominant cubic CEF on $3 \mathrm{H}_{6}$ ground multiplet with $J=6$ for $\mathrm{Tm}^{3+}$ ion. It is well known that the local field does not have cubic symmetry in the garnets. Nevertheless, Van Vleck has pointed out that the cubic approximation gives the right qualitative distinction between the behavior of the different ions in the garnets. We have adopted this Van Vleck theory [7]. Further, the notation of the crystal field Hamiltonian in the Lea, Leask and Wolf (LLW) theory [8] is used in this paper. The graphs of Lea et al. are expressed in terms of a potential

$$
V_{\text {cr }}=W\left\{\left[\frac{x}{F(4)}\right]\left[O_{4}{ }^{0}-5 O_{4}{ }^{4}\right]+\left[\frac{(1-|x|)}{F(6)}\right]\left[O_{6}{ }^{0}+21 O_{6}{ }^{4}\right]\right\} \text {. }
$$

The energy is measured in multiples of $W$ as a function of $x$, where $W$ and $x$ are given by

$$
V_{4} \beta F(4)=W x,
$$

and

$$
V_{6} \gamma F(6)=W(1-|x|)
$$

Here, $W$ acts like a scale factor and $x(-1.0 \leq x \leq+1.0)$ measures the ratio of fourth- to sixth-degree terms in the Hamiltonian. The parameters $W$ and $x$ have a different significance for different ions in terms of the basic constants $V_{4}, V_{6}$. In contrast to $W$ and $x$, the dimensionless ratio $V_{6} / V_{4}$ should not vary much from ion to ion, because $V_{6} / V_{4}$ is the measure of the relative importance of the fourth and sixth order harmonics. For example, the cubic symmetry gives the relation

$$
\frac{V_{6}}{V_{4}}=-\left(\frac{2}{7}\right)\left[\frac{\left\langle r^{6}\right\rangle_{4 f}}{\left\langle r^{4}\right\rangle_{4 f}}\right]\left(\frac{1}{R^{2}}\right)
$$

Here $R$ is the distance of the coordinating charges from the magnetic ion. Van Vleck has studied in detail and proposed the value for the late rare-earth garnets,

$$
\frac{V_{6}}{V_{4}}=-0.150
$$




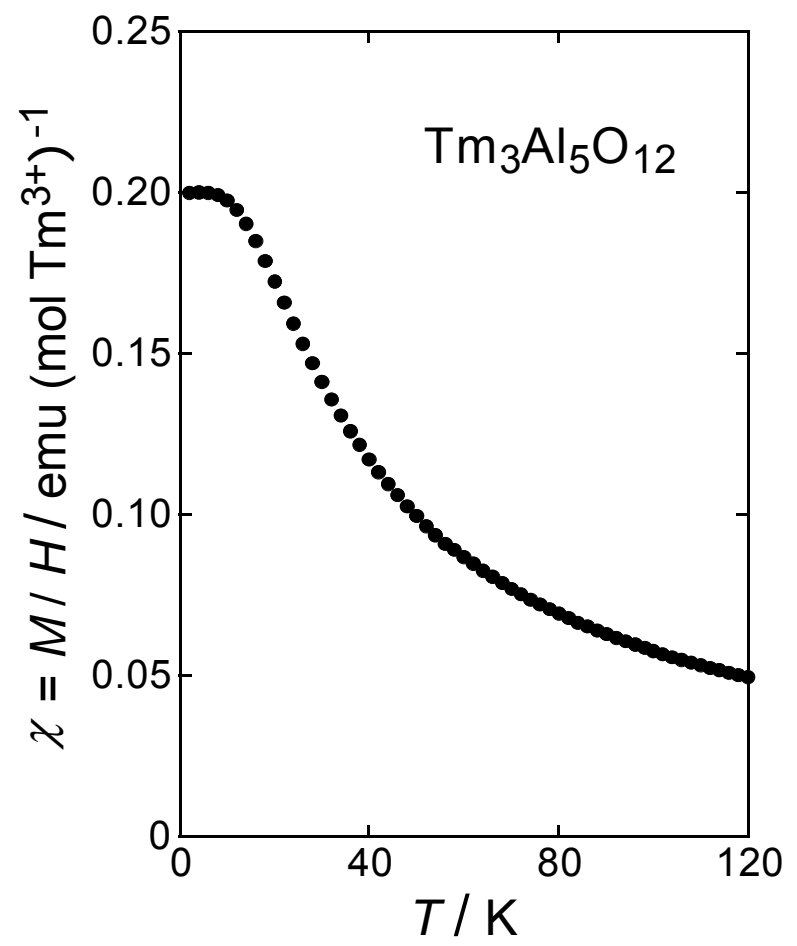

Fig. 3. Magnetic susceptibility on single crystal of $\mathrm{Tm}_{3} \mathrm{Al}_{5} \mathrm{O}_{12}$ per $\mathrm{mol} \mathrm{Tm}^{3+}$ ion as a function of temperature in a constant magnetic field $10.000 \mathrm{kOe}$.
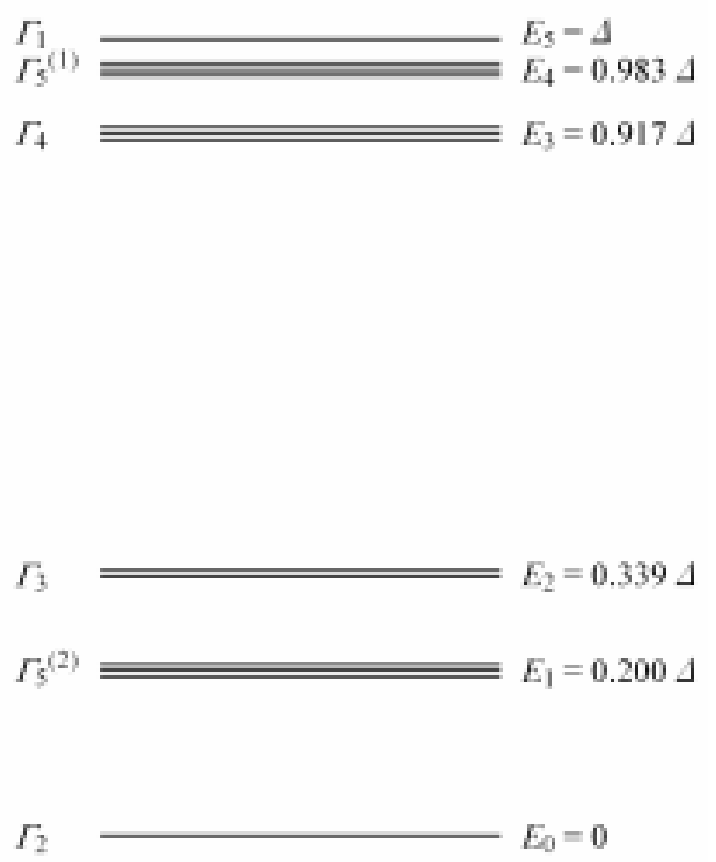

$$
\mathrm{Tm}^{3+} \text { : Ground multiplet }{ }^{3} \mathrm{H}_{6}(J=6)
$$

Fig. 4. The $4 f$ energy levels of $\mathrm{Tm}^{3+}$ ion due to the CEF. 
Table 1

Wave function of ${ }^{3} \mathrm{H}_{6}$ ground multiplet for $\mathrm{Tm}^{3+}$ ion in $\mathrm{Tm}_{3} \mathrm{Al}_{5} \mathrm{O}_{12}$.

\begin{tabular}{|c|c|}
\hline Energy & Wave function \\
\hline$E_{5}$ & $\left|\Gamma_{1}>=-0.6614\right|+4>+0.3536|0>-0.6614|-4>$ \\
\hline \multirow[t]{3}{*}{$E_{4}$} & $\left|\Gamma_{5}^{(1)}(a)>=-0.4556\right|+3>+0.3742|-1>+0.8077|-5>$ \\
\hline & $\left|\Gamma_{5}^{(1)}(b)>=-0.4556\right|-3>+0.3742|+1>+0.8077|+5>$ \\
\hline & $\left|\Gamma_{5}^{(1)}(c)>=-0.1452\right|+6>-0.6920|+2>+0.6920|-2>+0.1452 \mid-6>$ \\
\hline \multirow[t]{3}{*}{$E_{3}$} & $\left|\Gamma_{4}(d)>=+0.6846\right|+3>-0.4330|-1>+0.5863|-5>$ \\
\hline & $\left|\Gamma_{4}(e)>=+0.6846\right|-3>-0.4330|+1>+0.5863|+5>$ \\
\hline & $\left|\Gamma_{4}(f)>=-0.7071\right|+4>+0.0000|0>+0.7071|-4>$ \\
\hline \multirow[t]{2}{*}{$E_{2}$} & $\left|\Gamma_{3}(g)>=+0.5863\right|+6>+0.3953|+2>+0.3953|-2>+0.5863 \mid-6>$ \\
\hline & $\left|\Gamma_{3}(h)>=+0.2500\right|+4>+0.9354|0>+0.2500|-4>$ \\
\hline \multirow[t]{3}{*}{$E_{1}$} & $\left|\Gamma_{5}^{(2)}(i)>=+0.5692\right|+3>+0.8201|-1>-0.0589|-5>$ \\
\hline & $\left|\Gamma_{5}^{(2)}(j)>=+0.5692\right|-3>+0.8201|+1>-0.0589|+5>$ \\
\hline & $\left|\Gamma_{5}^{(2)}(k)>=-0.6920\right|+6>+0.1452|+2>-0.1452|-2>+0.6920 \mid-6>$ \\
\hline$E_{0}$ & $\left|\Gamma_{2}>=-0.3953\right|+6>+0.5863|+2>+0.5863|-2>-0.3953 \mid-6>$ \\
\hline
\end{tabular}


Table 2

Matrix elements $<\Gamma_{i}\left|J_{z}\right| \Gamma_{j}>$ in ${ }^{3} \mathrm{H}_{6}$ ground multiplet for $\mathrm{Tm}^{3+}$ ion in $\mathrm{Tm}_{3} \mathrm{Al}_{5} \mathrm{O}_{12}$.

\begin{tabular}{|c|c|c|c|c|c|c|c|c|c|c|c|c|c|}
\hline & $\left|\Gamma_{1}\right\rangle$ & $\left|\Gamma_{5}^{(1)}\right\rangle$ & $\left|\Gamma_{5}^{(1)}\right\rangle$ & $\left|\Gamma_{5}^{(1)}\right\rangle$ & $\left|\Gamma_{4}\right\rangle$ & $\mid \Gamma_{4}>$ & $\left|\Gamma_{4}\right\rangle$ & $\left|\Gamma_{3}\right\rangle$ & $\mid \Gamma_{3}>$ & $\left|\Gamma_{5}^{(2)}\right\rangle$ & $\left|\Gamma_{5}^{(2)}\right\rangle$ & $\left|\Gamma_{5}^{(2)}\right\rangle$ & $\left|\Gamma_{2}\right\rangle$ \\
\hline & & (a) & (b) & (c) & (d) & (e) & (f) & (g) & (h) & (i) & (j) & (k) & \\
\hline$<\Gamma_{1} \mid$ & 0 & 0 & 0 & 0 & 0 & 0 & 3.7414 & 0 & 0 & 0 & 0 & 0 & 0 \\
\hline$<\Gamma_{5}^{(1)}(\mathrm{a}) \mid$ & 0 & -2.7792 & 0 & 0 & -3.1415 & 0 & 0 & 0 & 0 & -0.8470 & 0 & 0 & 0 \\
\hline$<\Gamma_{5}^{(1)}(b) \mid$ & 0 & 0 & 2.7792 & 0 & 0 & 3.1415 & 0 & 0 & 0 & 0 & 0.8470 & 0 & 0 \\
\hline$<\Gamma_{5}^{(1)}(\mathrm{c}) \mid$ & 0 & 0 & 0 & 0 & 0 & 0 & 0 & -2.1158 & 0 & 0 & 0 & 0 & -0.9341 \\
\hline$<\Gamma_{4}(\mathrm{~d}) \mid$ & 0 & -3.1415 & 0 & 0 & -0.5000 & 0 & 0 & 0 & 0 & 1.6968 & 0 & 0 & 0 \\
\hline$<\Gamma_{4}(\mathrm{e}) \mid$ & 0 & 0 & 3.1415 & 0 & 0 & 0.5000 & 0 & 0 & 0 & 0 & -1.6968 & 0 & 0 \\
\hline$<\Gamma_{4}(\mathrm{f}) \mid$ & 3.7414 & 0 & 0 & 0 & 0 & 0 & 0 & 0 & -1.4142 & 0 & 0 & 0 & 0 \\
\hline$<\Gamma_{3}(\mathrm{~g}) \mid$ & 0 & 0 & 0 & -2.1158 & 0 & 0 & 0 & 0 & 0 & 0 & 0 & -4.6390 & 0 \\
\hline$<\Gamma_{3}(\mathrm{~h}) \mid$ & 0 & 0 & 0 & 0 & 0 & 0 & -1.4142 & 0 & 0 & 0 & 0 & 0 & 0 \\
\hline$<\Gamma_{5}^{(2)}(\mathrm{i}) \mid$ & 0 & -0.8470 & 0 & 0 & 1.6968 & 0 & 0 & 0 & 0 & 0.2821 & 0 & 0 & 0 \\
\hline$<\Gamma_{5}^{(2)}(\mathrm{j}) \mid$ & 0 & 0 & 0.8470 & 0 & 0 & -1.6968 & 0 & 0 & 0 & 0 & -0.2821 & 0 & 0 \\
\hline$<\Gamma_{5}^{(2)}(\mathrm{k}) \mid$ & 0 & 0 & 0 & 0 & 0 & 0 & 0 & -4.6390 & 0 & 0 & 0 & 0 & 3.6231 \\
\hline$<\Gamma_{2} \mid$ & 0 & 0 & 0 & -0.9341 & 0 & 0 & 0 & 0 & 0 & 0 & 0 & 3.6231 & 0 \\
\hline
\end{tabular}


By using $V_{6} / V_{4}=-0.150$, the ground state can be deduced to be $\Gamma_{2}$ singlet corresponding to

$$
\frac{F(4) \beta}{F(6) \gamma}=-0.2311, \quad W<0, \quad x=+0.606
$$

The reasonableness of this choice has been discussed by Van Vleck [7, 9]. We have calculated by using the value of $W<0, x=+0.600$ for $\mathrm{Tm}_{3} \mathrm{Al}_{5} \mathrm{O}_{12}$.

The LLW theory [8] leads to the energy level split of ${ }^{3} \mathrm{H}_{6}$ ground multiplet as shown in Fig. 4 and leads to the wave functions inherent in each energy level as is shown in Table 1, on the basis of $W<0, x=+0.600$. The energy level split is found from the bottom ground state to the highest state to be $\Gamma_{2}, \Gamma_{5}^{(2)}, \Gamma_{3}, \Gamma_{4}, \Gamma_{5}^{(1)}$ and $\Gamma_{1}$ The ground state is singlet $\Gamma_{2}$ with non-magnetic state. These wave functions in Table 1 consist of the mixing of different value of $J_{z}$ and each wave function is normalized.

Table 2 shows the matrix elements of $J_{z}$ for $\mathrm{Tm}^{3+}$ ion. Diagonal elements of this Table 2 cause the Curie-Weiss paramagnetic behavior and non-diagonal elements give rise to the Van Vleck temperature independent paramagnetic behavior

The general Van Vleck general formula for magnetic susceptibility is expressed as

$$
\begin{aligned}
\chi= & \frac{g_{J}^{2} \mu_{B}^{2}}{k_{B} T Z}\left[\sum_{i}\left|\left\langle\Gamma_{i}\left|J_{z}\right| \Gamma_{i}\right\rangle\right|^{2} \exp \left(-\frac{E_{i}}{k_{B} T}\right)\right] \\
& +\frac{2 g_{J}^{2} \mu_{B}^{2}}{Z}\left[\sum_{i, j}\left|\left\langle\Gamma_{i}\left|J_{z}\right| \Gamma_{j}\right\rangle\right|^{2} \frac{1}{E_{j}-E_{i}} \exp \left(-\frac{E_{i}}{k_{B} T}\right)\right], \\
Z= & \sum_{i} g_{i} \exp \left(-\frac{E_{i}}{k_{B} T}\right),
\end{aligned}
$$

where $\chi$ is the magnetic susceptibility per one ion, $g_{J}$ the Lande's $g$-factor, $\mu_{\mathrm{B}}$ the Bohr magneton, $k_{B}$ the Boltzman constant, $T$ the temperature, $J_{z}$ the $z$-component of the total angular momentum $J, Z$ the partition function, and $g_{\mathrm{i}}$ the degree of degeneracy. The explicit expressions for main part of first and second terms are described by, 


$$
\begin{aligned}
\sum_{i}\left|\left\langle\Gamma_{i}\left|J_{z}\right| \Gamma_{i}\right\rangle\right|^{2} \exp \left(-\frac{E_{i}}{k_{B} T}\right) \\
=0.1591 \exp \left(\frac{-E_{1}}{k_{B} T}\right)+0.5000 \exp \left(\frac{-E_{3}}{k_{B} T}\right)+15.4480 \exp \left(\frac{-E_{4}}{k_{B} T}\right), \\
\sum_{i, j}\left|\left\langle\Gamma_{i}\left|J_{z}\right| \Gamma_{j}\right\rangle\right|^{2} \frac{1}{E_{j}-E_{i}} \exp \left(-\frac{E_{i}}{k_{B} T}\right) \\
=\left(\frac{13.1268}{E_{1}-E_{0}}+\frac{0.8726}{E_{4}-E_{0}}\right) \exp \left(\frac{-E_{0}}{k_{B} T}\right) \\
+\left(\frac{13.1268}{E_{0}-E_{1}}+\frac{21.5207}{E_{2}-E_{1}}+\frac{5.7582}{E_{3}-E_{1}}+\frac{1.4348}{E_{4}-E_{1}}\right) \exp \left(\frac{-E_{1}}{k_{B} T}\right) \\
+\left(\frac{21.5207}{E_{1}-E_{2}}+\frac{2.0000}{E_{3}-E_{2}}+\frac{4.4764}{E_{4}-E_{2}}\right) \exp \left(\frac{-E_{2}}{k_{B} T}\right) \\
+\left(\frac{5.7582}{E_{1}-E_{3}}+\frac{2.0000}{E_{2}-E_{3}}+\frac{19.7375}{E_{4}-E_{3}}+\frac{13.9984}{E_{5}-E_{3}}\right) \exp \left(\frac{-E_{3}}{k_{B} T}\right) \\
+\left(\frac{0.8726}{E_{0}-E_{4}}+\frac{1.4348}{E_{1}-E_{4}}+\frac{4.4764}{E_{2}-E_{4}}+\frac{19.7375}{E_{3}-E_{4}}\right) \exp \left(\frac{-E_{4}}{k_{B} T}\right) \\
+\left(\frac{13.9984}{E_{3}-E_{5}}\right) \exp \left(\frac{-E_{5}}{k_{B} T}\right) \cdot .
\end{aligned}
$$

Consequently, the general formula of eq. (7) for the magnetic susceptibility includes only one unknown parameter $\Delta$ defined in Fig. 4 , therefore this value of $\Delta$ can be determined uniquely in comparison with the experimental result of the temperature dependence of the susceptibility. Here, Lande's $g$-factor is $7 / 6$ for $\mathrm{Tm}^{3+}$ ion and the anisotropy of $g$-factor is neglected in our treatment. The numerical calculation has been made to fit the experimental results, as indicated in Fig. 5. The characteristic feature of the temperature independent part is reproduced below $\approx 8 \mathrm{~K}$. There still remains the deviation of the intermediate temperature region over 20 to $200 \mathrm{~K}$, which may be attributed to the over simplification to the cubic symmetry. It is verified experimentally that the ground state is $\Gamma_{2}$ singlet. The value of $\Delta$ is obtained to be $339.5 \mathrm{~K}$. This magnitude of $\Delta$ determines the value of each energy level for CEF effect, as presented in Table 3. The energy separation between $\Gamma_{2}$ and the first excited state $\Gamma_{5}^{(2)}$ triplet is evaluated to be $68.0 \mathrm{~K}$.

It should be noted that $\mathrm{Tm}_{3} \mathrm{Al}_{5} \mathrm{O}_{12}$ exhibits a strong triplet-like absorption feature with peaks at $32.4,35.6$ and $39.3 \mathrm{~cm}^{-1}$. These energy separations have been found between the lowest and first excited triplet state $[10,11]$. Furthermore $28 \mathrm{~cm}^{-1}$ energy separations is observed in $\mathrm{Tm}^{3+}$ ion in $\mathrm{Y}_{3} \mathrm{Al}_{5} \mathrm{O}_{12}$ [12]. This small amount of energy separation is exactly related with our experimental result of $68.0 \mathrm{~K}$. The temperature dependence of ac magnetic susceptibility for $\left\{\operatorname{Tm}_{x} \mathrm{Y}_{1-x}\right\}_{3} \mathrm{Al}_{5} \mathrm{O}_{12}$ has been reported in Ref. [13].

Fig. 6 represents the $\Delta$ dependence of the magnetic susceptibility from the theoretical point 


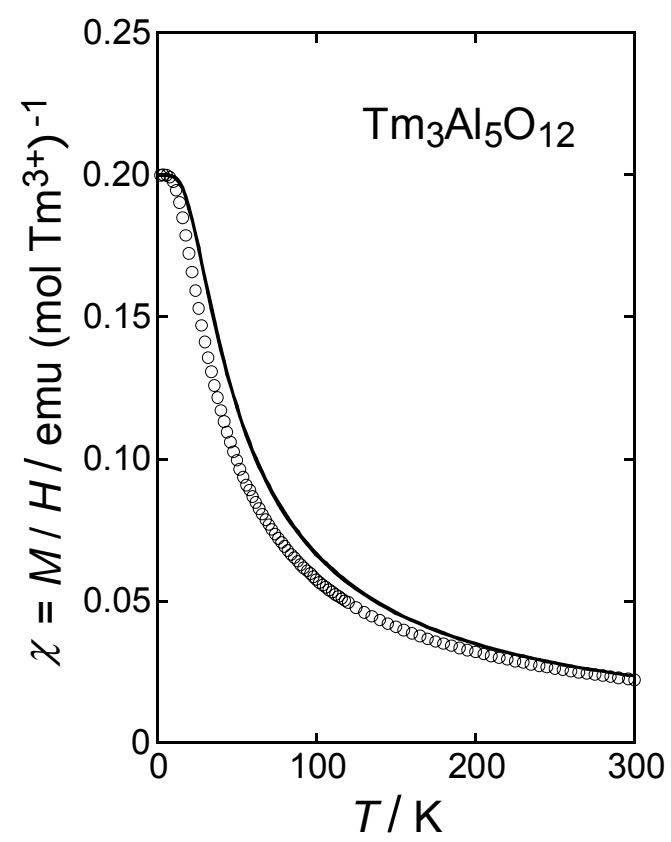

Fig. 5. Theoretical fitting magnetic susceptibility. The circle shows experimental result and the solid curve shows the calculated result with $\Delta=339.5 \mathrm{~K}$. Much emphasis is placed on the better fit to the low temperature data below $\approx 8 \mathrm{~K}$.

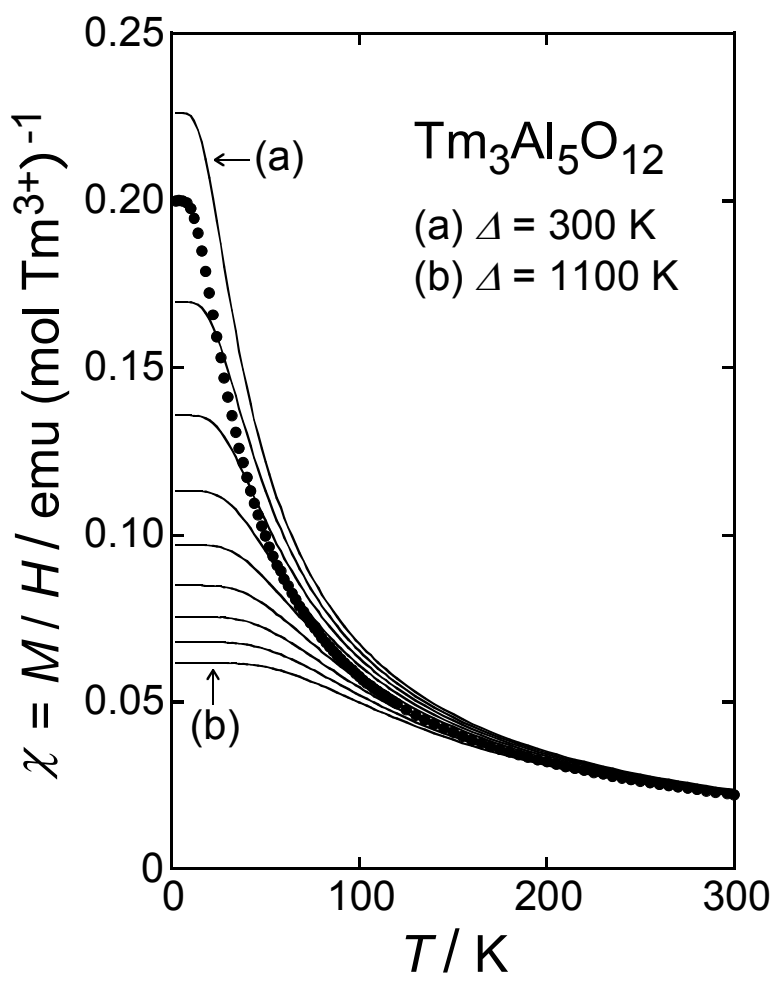

Fig. 6. The variation of the magnetic susceptibility on the magnitude of $\Delta$. The solid circle shows experimental result and the solid curves show the calculated results for various $\Delta$ at intervals of $100 \mathrm{~K}$ from (a) to (b). The value of $\Delta$ indicates the whole energy split in the CEF between $\Gamma_{2}$ and $\Gamma_{1}$ for ${ }^{3} \mathrm{H}_{6}$ ground multiplet of $\mathrm{Tm}^{3+}$ ion. 


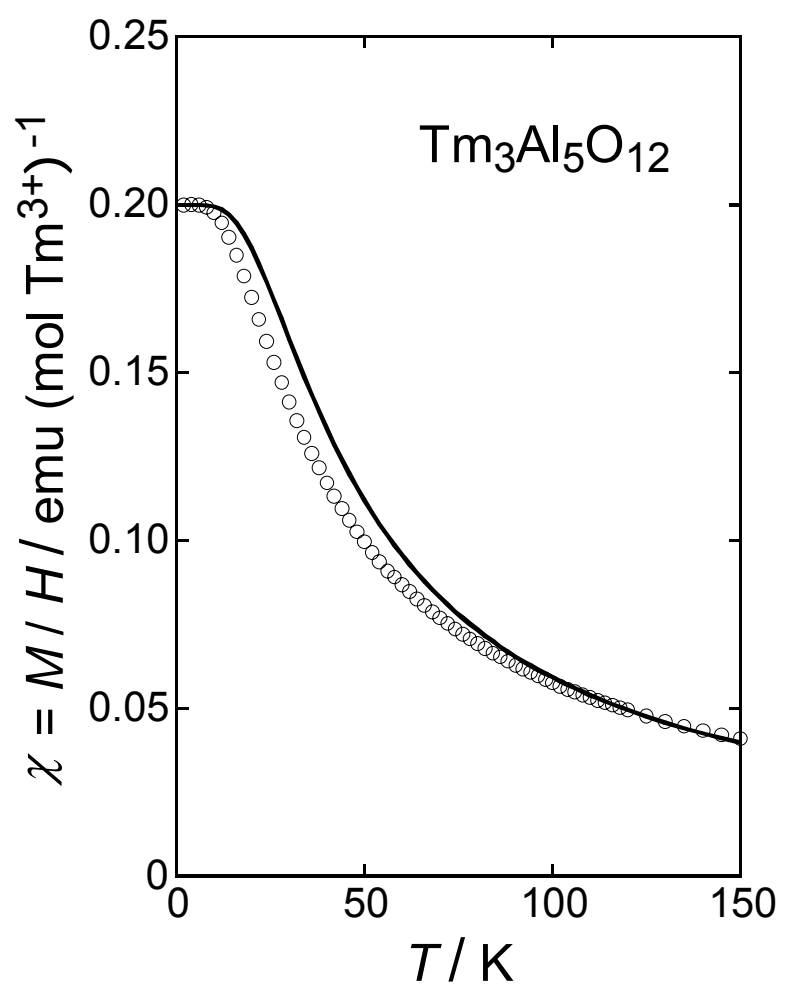

Fig. 7. A simplified model calculation that only low energy levels $\Gamma_{2}, \Gamma_{5}^{(2)}$ and $\Gamma_{3}$ are taken into account. The circle shows experimental result and the solid curve shows the calculated result. The energy difference between the ground state $\Gamma_{2}$ and the second exited state $\Gamma_{3}$ is $113.6 \mathrm{~K}$, see Table 3 .

Table 3

The values of energy level extracted from $\Delta=339.5 \mathrm{~K}$ for ${ }^{3} \mathrm{H}_{6}$ ground multiplet of $\mathrm{Tm}^{3+}$ ion in $\mathrm{Tm}_{3} \mathrm{Al}_{5} \mathrm{O}_{12}$.

\begin{tabular}{cl}
\hline State & Energy $(\mathrm{K})$ \\
\hline$\Gamma_{1}$ & 339.5 \\
$\Gamma_{5}^{(1)}$ & 333.6 \\
$\Gamma_{4}$ & 311.4 \\
$\Gamma_{3}$ & 115.2 \\
$\Gamma_{5}^{(2)}$ & 68.0 \\
$\Gamma_{2}$ & 0.0 \\
\hline
\end{tabular}


of view. There is an apparent characteristic: the higher the value $\Delta$, the higher is the beginning temperature below which the susceptibility becomes temperature independent, and thus the value itself of the temperature independent susceptibility is lower. In other word, the higher the value $\Delta$, the less is the ratio of the hybridization from the excited magnetic state to non-magnetic ground state, therefore the magnitude of the susceptibility at $0 \mathrm{~K}$ becomes lower.

In addition, a simplified model calculation is demonstrated in Fig. 7. Here only lower energy band due to $\Gamma_{2}, \Gamma_{5}^{(2)}$ and $\Gamma_{3}$ is taken into accounted for this calculation. The basic feature is reproduced by this model below $150 \mathrm{~K}$. The difference of the value of $\Gamma_{3}$ energy between Figs. 5 and 7 is negligibly small as listed in Table 3.

\section{References}

[1] J. A. Koningstein, C. J. Kane-Maguire, Can. J. Chem. 52 (1974) 3445.

[2] W. P. Wolf, M. Ball, M. T. Hutchings, M. J. M. Leask, A. F. G. Wyatt, J. Phys. Soc. Jpn 17, (Supplement B-1) (1962) 443.

[3] S. C. Abrahams, S. Geller, Acta Cryst. 11 (1958) 437.

[4] S. Geller, Z. Krist, 125 (1967) 1 and references therein.

[5] S. Nagata, H. Sasaki, K. Suzuki, J. Kiuchi, N. Wada, J. Phys. Chem. Solids 62 (2001) 1123 and references therein.

[6] J. Awaka, T. Kurimoto, S. Nagata, Physica B 329-333 (2003) 669.

[7] M. M. Schieber, C. C. Lin, J. H. Van Vleck, J. Phys. Chem. Solids 27 (1966) 1041.

[8] K. R. Lea, M. J. M. Leask, W. P. Wolf, J. Phys. Chem. Solids 23 (1962) 1381.

[9] J. H. Van Vleck, J. Phys. Chem. Solids 27 (1966) 1047.

[10] R. C. Milward, Phys. Lett. 25A (1967) 19.

[11] R. W. Bierig, L. Limai, J. Appl. Phys. 36 (1965) 1199.

[12] G. A. Slack, D. W. Oliver, Phys. Rev. B 4 (1971) 592 and references therein.

[13] D. D. Perlov, A. A. Sorokin, V. A. Feodorov, A. P. Dodokin, J. Mag. Mag. Mater. 123 (1993) 187. 